\title{
Números binários em livros didáticos de matemática e de computação: uma comparação
}

Herman do Lago Mendes herman2000@zipmail.com.br 0000-0001-5631-1578

Secretaria de Estado da Educação de Sergipe

\section{RESUMO}

Identificando a abordagem de números binários em livros didáticos de computação e de matemática do Ensino Fundamental, objetiva-se comparar essa abordagem a partir de praxeologia e de seus critérios de avaliação (elementos da Teoria Antropológico do Didático). Primeiro se faz a comparação por meio de elementos praxeológicos: tipos de tarefa, técnica, tecnologia e teoria. Depois se faz a comparação por meio de critérios de avaliação praxeológica: critério de razão de ser, critério de relevância, critério de identificação, critério prático-técnico, critério tecnológico-teórico e critério de relação com outros saberes. Conclui-se que a abordagem de números binários no livro didático de computação analisado é mais ampla e aprofundada do que nos livros didáticos de matemática do Ensino Fundamental avaliados pelo PNLD 2014. Há, contudo, alguns elementos em comuns.
\end{abstract}

PALAVRAS-CHAVE: Números binários. Livro didático de matemática. Livro didático de computação. 


\section{INTRODUÇÃO}

Segundo Mendes (2014), os números binários são abordados em livros didáticos de matemática do Ensino Fundamental avaliados pelo Programa Nacional do Livro Didático (PNLD 2014) por meio de dois blocos de conteúdo da matemática básica: Grandezas e Medidas, e Números e Operações. O primeiro bloco de conteúdo estuda as unidades de medida da informática (bytes, megabytes, gigabytes, etc.) e o segundo bloco de conteúdo estuda o sistema de numeração binário.

A partir da análise praxeológica de livros didáticos de matemática (LDMAT) avaliados pelo PNLD 2014 por Mendes (2015, 2017a, 2017b), identificamos os seguintes temas e assuntos referentes ao estudo de números binários: unidades de memória de computadores (byte, megabyte, gigabyte, etc.) e conversõesentre números representados nos sistemas de numerações binário e decimal por meio de duas técnicas diferentes: representação de um número natural por meio de uma soma de potências de base 2 e por meio de divisões sucessivas por 2 .

A partir da análise praxeológica de livro didático de ciência da computação (LDCC) por Mendes (2016a), identificamos assuntos, temas e domínios referentes ao estudo de números binários: converter um número representado no sistema binário em um número representado no sistema decimal, codificação e compressão de dados (palavras, números e imagens), detecção e correção de erros de números de código (binário) e teoria da informação.

A partir dos resultados de pesquisa de Mendes (2014, 2015, 2016a, 2016b, 2017a, 2017b), é percebido que existe abordagem de estudo de números binários tanto em LDMAT do Ensino Fundamental aval iados pelo PNLD 2014, como também há abordagem de estudo de números binários em LDCC para esse mesmo nível de ensino; procuramos responder os seguintes questionamentos de pesquisa: Os números binários são abordados de maneira igual, semelhante ou diferente em livros didáticos de matemática e de computação? Caso a abordagem de estudo de números binários seja diferente nesses livros didáticos, quais são essas diferenças? Que áreas do saber (computação ou matemática) os números binários são mais aprofundados (tratando-se dos livros didáticos)? Quais são os temas e áreas científicas relacionados, auxiliados ao estudo de números binários?

Vale destacar que os números binários surgiram na ciência Matemática em $1600^{1}$, mas que ressurgiu no final da década de 1940: como conteúdo de estudo escolar, nos Estados Unidos, a partir da construção de computadores eletrônicos e de calculadoras digitais; e por meio da teoria matemática da comunicação de Claude Shannon (GLASER, 1981).

Os números binários são um objeto de saber que contribui atual mente à "Era Digital" devido ao advento da digitalização mundial a partir do século XX. A presença de aspectos relativosà representação binária de número tem aumentado em consequência a sua utilização indispensável na comunicação entre artefatos

1 Glaser (1981) afirma que antes de 1951, quando J.W. Shirley apresentou os manuscritos publicados de Hariot (em 1600), estavam a mbos Leibniz (em 1703) e o bispo Juan Caramuel e Lobkowitz (em 1670) como criadores do sistema binário. Assim, Thomas Hariot foi a primeira pessoa a registrar o uso do sistema binário. 
tecnológicos digitais por meio de sequências de 0s e 1s em codificações de caracteres, figuras, vídeos, sons, entre outros.

O sistema de numeração binário utiliza apenas dois dígitos, 0 e 1, para representar o número. Exemplo: o número 15 (quinze) representado no sistema decimal precisou de 2 dígitos diferentes, 1 e 5 , para escrever o número ou seja, 15 $=1.10^{1}+5.10^{\circ}=10+5$ (1 dezena mais 5 unidades); o número 1001 (um zero zero um) é representado no sistema binário por apenas 2 dígitos diferentes: 0 e 1 ou seja, é $1001=1 \cdot 2^{3}+0.2^{2}+0.2^{1}+1 \cdot 2^{0}$. Daí temos que 15 (quinze) na base 10 é igual a 1001 (um zero zero um) na base 2 . Ou seja, os números são os mesmos, porém podem ser representados de maneiras diferentes, por símbolos diferentes: 15 (quinze) e 1001 (um zero zero um).

Objetivamos comparar a abordagem de números binários em LDMAT e em LDCC, baseando-nos nos trabal hos de Mendes (2015, 2016a, 2017a, 2017b). Para tal, utilizamos a praxeologia e seus critérios de avaliação - elementos teóricos da Teoria Antropológico do Didático (TAD) - como ferramenta de análise de pesquisa.

\section{PRAXEOLOGIA}

Segundo Bosch e Gascón (2007), qualquer fazer, ação humana existe (implicitamente ou explicitamente) pelo me nos uma justificativa e explicação para tal. Ou seja, a TAD defende uma tese de que qual quer que seja o fazer humano, ao menos, ele é questionado, explicado (parcialmente), executado (de maneira intencional e inteligível), por alguma justificativa ou explicação; a TAD defende princípios inter-relacionados de elementos práticos e teóricos, imbricados, na ação humana. Essa imbricação é definida na TAD como praxeologia ou organização praxeológica.

A partir da etimologia, a palavra praxeologia deriva de duas palavras gregas, práxis e logos. Práxis significa a parte prática da ação humana. Logos significa a parte lógica, racional e inteligível (teórica) dessa prática humana. Praxeologia é composta por quatro Ts: tarefa, técnica, tecnologia e teoria.

Tarefa ou tipo de tarefa está relacionado ao fazer coisas. Geralmente expressa por um verbo: cortar o cabelo, comer bolo, codificar um texto; é uma ação, obra humana intencionada (CHEVALLARD, 1998a); requer um propósito relativamente específico (CHEVALLARD, 1998b). Exemplo: converter o número 10101 representado no sistema de numeração binário em um número representado no sistema decimal.

Técnica está relacionada ao como fazer, executar as tarefas ou tipos de tarefas; é a "arte" da ação humana; é o procedimento de real ização do tipo de tarefa. Vale destacar que a técnica, em seu procedimento de execução do tipo de tarefa, é formada também, por tarefas, etapas. Estas são chamadas subtarefas (CHEVALLARD, 1998a). Exemplo de técnica: primeiro, desenhar pontos em apenas uma face do cartão de tal maneira que cada cartão contenha quantidades, distintas, em potências de base $2(1,2,4,8,16,32$, etc.) (subtarefa). (Figura 1); 


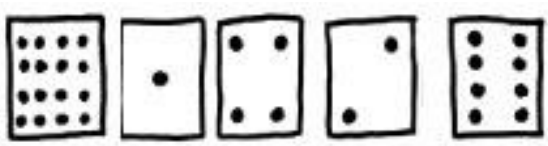

Segundo, posicionar cada cartão, em ordem crescente, da direita para a esquerda (subtarefa) (Figura 2);

Figura 2 - Cartões em ordem crescente da direita para a esquerda

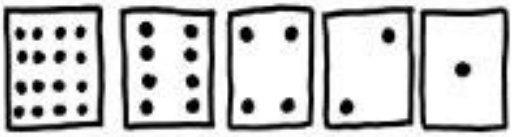

Terceiro, posicionar cada dígito do número binário a ser convertido em decimal a baixo de cada cartão (subtarefa) (Figura 3);

Figura 3 - Cada dígito a baixo de cada cartão

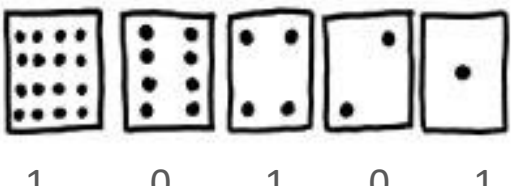

Quarto, corresponder os dígitos $1 \mathrm{~s}$ com a face do cartão pontuado e corresponder os dígitos 0s com a face do cartão não pontuado ou virar, apenas, a face do cartão que não contém pontos que correspondem ao dígito 0 (subtarefa) (Figura 4);

Figura 4 - Virar os cartões que tem dígito zero

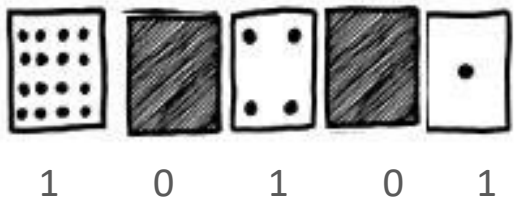

Ultimo, somar os pontos de cada cartão que correspondem a 1 (subtarefa). 0 total (21) será o número, convertido para o sistema decimal, procurado: $16+4+1$ $=21$

Segundo Chevallard (1998a, 1998b), o tipo de tarefa e a técnica, ambas, constituem a práxis. Esses dois elementos da praxeologia é definido como bloco prático-técnico.

Tecnologia é a explicação ou justificativa, de maneira racional e lógica, da veracidade da técnica. Exemplo: Por que expomos os cartões da direita para a esquerda?

Organizando os cartões da direita para a esquerda conforme a ordem crescente da quantidade de pontos em potências de base 2, a partir de 1, configura-se dessa maneira, as ordens numéricas binárias. Define, portanto, o sistema de numeração binário. 0 1으 cartão contém 1 ponto, o 2o cartão (lado esquerdo do 1ㅇ) contém 2 pontos, o 3 o cartão (lado esquerdo do 2ㅇ) contém 4 pontos, o 4ㅇ cartão (lado esquerdo do 3ㅇ) contém 8 pontos e assim sucessivamente. Ou seja, a 1aa 2ạa , 3ạ, n-ésima ordem numérica binária mantém 
correspondência ao 1 을 cartão, ao 2 o cartão, ao 3 o cartão, ao n-ésimo cartão (MENDES, 2016a, p.321).

Teoria é o maior nível de explicação das tecnologias; é proveniente de provas, de conceitos, de discurso mais formal, abstrato. Exemplo: Podemos, então, converter qualquer número representado no sistema binário em um número representado no sistema decimal? Qualquer número natural $\mathrm{m}$ pode ser representado em uma base $b \neq 1$, também natural não nula da seguinte maneira:

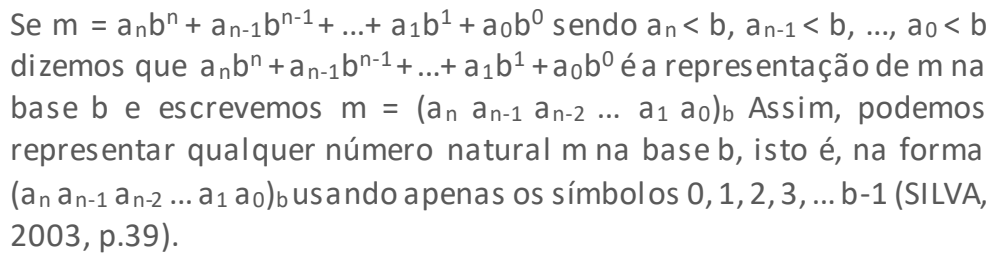

Portanto, temos que $(10101)_{2}=1 \cdot 2^{4}+0.2^{3}+1 \cdot 2^{2}+0.2^{1}+1.2^{0}=(21)_{10}$

Segundo Chevallard (1998a, 1998b), a tecnologia e a teoria, ambas, constituem o logos. Esses dois elementos da praxeologia é definido como bloco tecnológico-teórico.

Chevallard (1998b) define quatro categorias praxeológicas: praxeologia pontual, praxeologia local, praxeologia regional e praxeologia global. Todas estas apresentam, a princípio, quatro elementos praxeológicos. Embora Chevallard (ibidem) nos alerte de que a depender da instituição (livro didático, sala de aula, colégio, etc.) que esteja trabal hando o objeto do saber, pelo menos um elemento praxeológico pode estar ausente.

Praxeologia pontual apenas mantém relação com um único tipo de tarefa, uma técnica, uma tecnologia e uma teoria.

Praxeologia local apenas leva em consideração uma única tecnologia. Ou seja, há apenas uma única justificativa ou explicação para a veracidade de pelo menos uma técnica executada para um tipo de tarefa.

Praxeologia regional apenas leva em consideração uma única teoria. Ou seja, há apenas uma única teoria para pelo menos uma tecnologia, várias técnicas e tipos de tarefas mantém relação com essa única teoria.

Praxeol ogia gl obal é a agregação de várias praxeologias regionais relacionadas a várias teorias.

A organização praxeológica (matemática ou computação, etc.) parte de um tratamento didático para/com a sua organização, planejamento, seleção, discussão de métodos, técnicas e abordagem. Ou seja, existem preocupações didáticas que giram em torno dessas praxeologias. Portanto, a praxeologia didática ou organização praxeológica didática é atrelada àquelas organizações praxeológicas. Ela pode (re)formular elementos praxeológicas matemática, computação, etc. É a partir desse entendimento que iniciamos a análise comparativa praxeológica matemática e praxeológica computação entre LDMATe LDCC, referente ao estudo de números binários, para em seguida compará-los por meio de praxeologia didática (critérios de avaliação praxeológica). 
Os critérios de avaliação praxeológica giram em torno de questionamentos comuns frente ao objeto de saber (números binários) analisado (CHEVALLARD, 1998b):

Critério de razão de ser: Quais são as razões para estudar o objeto do saber (números binários)? As tarefas fazem parte do cotidiano dos estudantes?

Critério de relevância: Por que estudar esse objeto do saber? Qual é a importância de se estudar esse objeto do saber?

Critério de identificação: Existem quantos tipos de tarefas, técnicas, tecnologias e teorias? Que tipo de praxeologia a coleção pode ser configurada?

Critério prático-técnico: Os tipos de tarefas são executados por técnicas? As técnicas são ilustradas, exercitadas? Por que ensinar essa técnica e não outra?

Critério tecnológico-teórico: As técnicas são justificadas e explicadas? Por que a técnica funciona? Existe algum embasamento teórico que prove a veracidade das técnicas trabalhadas?

Critério de relação com outras áreas de saber ou outros blocos de conteúdos: Quais são os pré-requisitos para se estudar determinado objeto do saber? Quais são as disciplinas ou áreas de saber envolvidas na aprendizagem de determinado saber?

\section{METODOLOGIA}

A partir da identificação de números binários em LDMAT e em LDCC por Mendes (2014, 2015, 2016a, 2016b, 2017a, 2017b), iniciamos a comparação da abordagem de números binários em livros didáticos de matemática e de computação a partir de organizações praxeológicas matemática e computação: tipos de tarefas, técnicas, tecnologias e teorias. Em seguida, continuamos a comparação, mas a partir de critérios de avaliação praxeológica (organizações praxeológica didática): critério de razão de ser, critério de relevância, critério de identificação, critério prático-técnico, critério tecnológico-teórico, e critério de relação com outras áreas de saber ou de outros blocos de conteúdos. Definimos cada elemento praxeológico como uma seção.

Esta pesquisa é bibliográfica, do ponto de vista teórico e metodológico, utiliza a praxeologia e seus critérios de avaliação, ambos, elementos da TAD.

COMPARAÇÃO DE ORGANIZAÇÕES PRAXEOLÓGICAS (MATEMÁTICA E COMPUTAÇÃO) REFERENTE AO ESTUDO DE NÚMEROS BINÁRIOS

\section{TIPOS DE TAREFAS}

Mendes (2016a) identificou vinte tipos de tarefas em LDCC enquanto que Mendes (2017a) identificou doze tipos de tarefas em LDMAT.

Tipos de tarefas comuns em LDMAT e LDCC referentes ao estudo de sistema binário: converter um número representado no sistema binário em um número 
representado no sistema decimal; representar um número natural como uma soma de potências de base 2 e determinar a capacidade de memória de um computador.

Os LDMAT solicitam tarefas de unidades de medida de informação, kilobyte, megabyte, gigabyte, terabyte dentre outras unidades, e de unidades de medida de processamento de dados, megahertz e gigahertz, enquanto que LDCC recorreu apenas à definição do bit e do byte como unidades de medida de informação.

Nos LDMAT existiu a solicitação de conversões entre unidades de medida da informática, enquanto que esta conversão não foi solicitada no LDCC. No entanto, o LDCC solicitou tipos de tarefas não solicitadas em LDMAT: identificar padrões ou sequências; explicar elementos conceituais de sistema de numeração binário; contar por meio do sistema binário; criar uma técnica para escrever um número natural como composição de potências de base 2; e tarefas referentes à codificação/decodificação, compressão, detecção e correção de erros de dados e medir informação por meio da noção teórica de Shannon.

A subtarefa mais presente em LDCC é: representar dados por dígitos binários. Já em LDMAT, as subtarefas mais abordadas são: converter unidades de medida de mesma grandeza, que ora foi considerado aqui como tipo de tarefa, ora como subtarefa, a depender de sua abordagem no livro ao solicitar um exercício ou um problema; e representar números das medidas de informação como potências de base 2 (utilização da potenciação).

\section{TÉCNICAS}

Mendes (2015, 2017a) identificou em LDMAT três técnicas referentes às conversões de números representados entre o sistema binário e o sistema decimal (vice-versa). Já em LDCC, Mendes (2016a) identificou, apenas, uma técnica para converter números representados no sistema binário em um número representado no sistema decimal.

Não existe ilustração e nem exercitação de técnicas de conversões entre unidades de medida da informática em LDCC (MENDES, 2016A) e em LDMAT (MENDES, 2015, 2017a). Embora Mendes (2015, 2017a) tenha implementadouma técnica de conversão entre unidades de medida da informática a partir da investigação de (duas) técnicas referentes às unidades de medida de mesma grandeza (capacidade) em volume diferente do identificado por unidades da informática.

Mendes (2016a) identificou duas técnicas de codificação/decodificação de imagens (preto e branca, e colorida) e uma técnica de compressão de textos, duas técnicas de detecção e correção de erros de códigos binários e uma técnica para medir informação. Apesar de uma coleção de LDMAT mostrar uma aplicação de números binários por meio da criação de um código de barras, nenhuma técnica referente à representação de dados é trabalhado (MENDES, 2017a).

\section{TECNOLOGIAS}


Ambos LDCC e LDMAT explicam que 1 bit é a menor unidade de informação e que pode assumir, apenas, dois valores: 0 ou 1 . O byte corresponde a 8 bits. No entanto, os LDMAT ora definema base 10, ora definema base 2 numérica em medidas de informação. Ambas bases numéricas mantêm as mesmas nomenclaturas e partes literais do sistema métrico decimal: quilobyte (KB), megabyte (MB), gigabyte (GB), etc. Ou seja, na medida de informação,

O número é representado por potências de base $2\left(2^{10}, 2^{20}, 2^{30}, 2^{40}, 2^{50}, 2^{60}\right)$ e a parte literal é definida por símbolos próprios: $\mathrm{KiB}, \mathrm{MiB}, \mathrm{GiB}, \mathrm{TiB}, \mathrm{PiB}, \mathrm{EiB}$, etc. Os nomes e símbolos dos prefixos correspondentes a essas potências de 2 são respectivamente: kibi e Ki; mébi e Mi; gibi e Gi; tébi e Ti; pébi e $\mathrm{Pi}$; exbi e Ei (MENDES, 2016b, pp.213, 214).

Em LDMAT as unidades de medida de informação, kilobyte, megabyte, gigabyte, entre outras, são enquadradas como múltiplos do byte e que fazem parte do sistema métrico decimal; as velocidades de processamento de dados, megahertz e gigahertz, são definidas por ciclos por segundos.

As demais justificativas e explicações das técnicas em LDCC giram em torno de como podemos representar textos e imagens a partir de números binários e de como armazenar dados em memória de computador.

\section{TEORIAS}

Apenas uma coleção de LDMAT apresenta noções teóricas de grandezas e de medidas, as demais não apresentam elementos teóricos (MENDES, 2017a). Os LDCC não abordam sobre essas noções teóricas, embora mantenha noções teóricas de medida de informação de Claude Shannon.

\section{COMPARAÇÃO DE CRITÉRIOS DE AVALIAÇÃO PRAXEOLÓGICA (MATEMÁTICA E COMPUTAÇÃO) REFERENTE AO ESTUDO DE NÚMEROS BINÁRIOS}

\section{CRITÉRIO DE RAZÃO DE SER}

Os LDMAT abordam as unidades de medida da informática (armazenamento e processamento de dados) porque constituem um saber social atual de caráter prático e relacional aos outros saberes e áreas de conhecimento. A partir do advento da informática nos dias atuais, o estudante pode perceber a utilidade das grandezas e medidas - medidas da informática - no seu dia-a-dia. Assim, a razão das unidades de medida da informática serem estudados em LDMAT gira em torno de sua aplicabilidade social; Números binários configuram-se como assunto de suporte ao estudo de potenciação ou curiosidade no Manual do Professor. Já em LDCC as unidades de medida de informação, bit e byte, e os números binários são estudados porque são pré-requisitos fundamentais e necessários para a aprendizagem de outros saberes da Computação: técnicas de compressão, codificação/decodificação, deteç̧ão e correção de erros de dados e medição de informação. Além disso, os computadores utilizam números binários para representar informações. Assim, a razão dos números binários serem estudados em LDCC é intracomputação. Ou seja, a computação recorre de questionamentos 
e saberes dela própria para respondê-las. Portanto, a razão dos números binários serem estudados em livros didáticos de LDCC e de LDMAT é distinta: quase todos os LDMAT (exceto uma coleção, que planeja estudar unidades de medida da informática em primeiro plano) planejam o estudo de números binários como saber social ou curiosidade ou auxiliar ao estudo de potenciação. Enquanto que em LDCC os números binários constituem o lócus de conhecimento da ciência da computação.

\section{CRITÉRIO DE RELEVÂNCIA}

Em LDMAT, o armazenamento e processamento de dados de computador são decorridos graças às unidades de medida em informática; o estudo de números binários pode auxiliar a compreensão de potenciação (assunto da Matemática). Já em LDCC o estudo de números binários não é mantido como assunto auxiliar a compreensão de potenciação, mas o seu estudo é fundamental e necessário para a realização de técnicas de medição de informação, de codificação/decodificação, detecção/correção de erros e compressão de dados (objetos do saber de Computação). Além disso, apresentam justificativas, contextualizadas, situações atuais do cotidiano dos estudantes sobre o porquê estudar nú meros binários. Exemplos: "Quando os dados devem ser transmitidos por uma linha telefônica ou enlace de rádio, tons de alta e baixa frequência são utilizados para os zeros e uns" (BELL et al, 2011, p.12);

Imagine que você está depositando $\mathrm{R} \$ 10,00$ em dinheiro na sua conta bancária. O caixa digita o valor do depósito e o envia para um computador central. Contudo, suponha que alguma interferência ocorreu na linha enquanto o montante está sendo enviado e o código de $R \$ 10,00$ é alterado para $R \$ 1.000,00$. [...] Portanto, um computador precisa verificar que os dados recebidos não foram corrompidos por algum tipo de interferência elétrica na linha de transmissão [...] Colocando bits em linha e colunas imaginárias, e acrescentando bits de paridade para cada linha e coluna, podemos não apenas detectar se ocorreu um erro, mas quando este erro aconteceu. O valor do bit incorreto é modificado e, com isso, realizamos sua correção (BELL et al, 2011, p.36).

\section{CRITÉRIO DE IDENTIFICAÇÃO}

Os LDMAT configuram-se como praxeologia pontual ou praxeologia local. No geral, Mendes (2017a) identificou doze tipos de tarefas; três técnicas claras e bem identificadas referentes às conversões de números representados nos sistemas binário e decimal e duas técnicas implícitas, referentes às conversões de unidades de medida da informática; apenas uma tecnologia (superficial) referente a estas unidades de medida e um princípio de teoria (grandeza e medida). Em comparação com a abordagem de números binários entre LDMATe LDCC, este é mais amplo e aprofundado do que os outros porque Mendes (2016a) identificou vinte tipos de tarefas; sete técnicas claras e bem identificadas: uma técnica referente a conversão de um número representado no sistema binário em sistema decimal, uma técnica de medição de informação, duas técnicas de codificação/decodificação, duas técnicas de detecção/correção de erros de dados 
e uma técnica de compressão de dados. Todas as técnicas foram justificadas e explicadas e uma noção teórica foi empregada para medir informação.

\section{CRITÉRIO PRÁTICO-TÉCNICO}

As tarefas ou tipos de tarefas presentes em LDMAT são organizadas em seções próprias: "Atividades", "Exercícios e problemas", "Revisão", "Testes", etc; são apresentadas após uma breve explicação sobre o assunto. Em relação ao LDCC, a exploração de tarefas é realizada na "Folha de Atividade". Esta é iniciada por objetivo, problemática e por elementos de motivação ao desenvolvimento da atividade, seguida por sua justificação. Nas instruções existem perguntas que direcionam ou destacam elementos pertinentes aos conceitos e contextos da computação. As folhas de atividades abordam sobre questão do posicionamento das ordens numéricas binárias; destaca o bit (menor unidade de informação) representado por um cartão (contendo ou não pontos) e a partir disso, trabalha as conversões entre números representados nos sistemas de numerações binários e decimais; trabalha os processos conceituais de codificação/decodificação por meio de representação de códigos binários de imagens, preta e branca, e colorida. Já em LDMAT, ora as unidades de memória de computadores (byte, quilobyte, gigabyte) são estudadas em primeiro plano, ora são estudadas em segundo plano, definindo potenciação como tema principal; são contextualizadas por elementos da informática: backup, download, computador, etc.

Os tipos de tarefas presentes em LDMAT são claramente expostos e bem identificados, diferentemente das técnicas de conversão entre unidades de medidas de mesma grandeza (medida de informação e medida de velocidade de processamento de dados). Apenas as técnicas de conversão entre números representados nos sistemas de numerações binário e decimal são identificados claramente. Diferentemente identificado em LDCC que todas as tarefas e técnicas são claramente expostas e bem identificadas.

Apenas uma coleção de LDMAT ilustra no Manual do Professor três técnicas de conversões entre números representados no sistema decimal e no sistema binário (vice-versa). No entanto, apenas é solicitada a execução de um tipo de conversão (execução de uma tarefa) pelo estudante: verificar a conversão do número representado no sistema binário em um número representado no sistema decimal. Esta tarefa também se configura como tipo de tarefa em LDCC.

Identificamos duas técnicas de conversão de um número representado no sistema de numeração decimal em um número representado no sistema de numeração binário em LDMAT: técnica das divisões sucessivas por 2 e técnica da representação do número representado no sistema decimal por meio de uma expressão polinomial numérica de potências de base dois, simplesmente, ou recorrendo à uma tabela contendo grupos de potências de 2. Este tipo de conversão "decimal em binário" não é trabal hado em LDCC. Embora, a técnica de conversão de um número representado no sistema binário em um número representado no sistema decimal o seja trabal hado em ambos LDMAT e LDCC. No entanto, a técnica utilizada é diferente. Em LDMAT, a técnica "binário em decimal" se baseia na escrita de uma expressão polinomial numérica de potências de base dois recorrendo-se a uma tabela de grupos de potências de 2 ou ordens do sistema 
binário. Enquanto que em LDCC, a técnica "binário em decimal" se baseia nas correlações entre dígitos binários (1 e 0) e objetos dicotômicos (lados de um cartão, lâmpada de Natal acessa e apagada, sons grave e agudo (bip e bop), dedos das mãos levantados e abaixados). Especificamente, temos a organização de cartões pontuados, ou não, que corresponde o dígito 1 à uma face do cartão pontuado e corresponde o dígito 0 à outra face do cartão não pontuado. A partir dessa regra, a face do cartão que corresponder ao dígito 0 , deverá ter cartão virado, mudando-se a face de cima para baixo...

Identificamos sete técnicas no LDCC, tal que elas são trabalhadas nas "Folhas de Atividades" ou no próprio corpo de cada seção. Ora são articuladas ou/e complementadas ou aprofundadas no próprio enunciado das tarefas por meio de gerenciamento de perguntas, ora são trabalhadas após a ilustração de técnicas.

As unidades de memória de computadores em LDMAT trabalham os múltiplos de byte (quilobyte, megabyte, gigabyte, terabyte, petabyte, exabyte e zetabyte), enquanto que o LDCC não trabalham os múltiplos do byte.

A respeito do momento de trabalhar a técnica referente às unidades de medida de informação, é inexistente em todos os livros didáticos de matemática analisados. Em relação às técnicas de conversões entre números representados nos sistemas de numerações binário e decimal, apenas são ilustradas pelos autores, não existindo, portanto, momento de sua exercitação pelos estudantes.

Baseado no entendimento de que a avaliação é um conjunto de ações cujo objetivo sempre remete a performance e melhoramento de determinado trabalho por meio de observação, de análise, de discussão, de reflexões, entre outras instâncias, dos processos de ensino e de aprendizagem; o momento de avaliação presente nos LDMAT mantém as seguintes propostas (somadas ao momento de trabalhar a técnica): pesquisas referentes àquelas unidades de medida na informática, trabalho em grupo e em pesquisa por meio das ativida des de códigos de barras e do Jogo do Nim, por exemplo, e/ou resolução de exercícios e de problemas. Já em LDCC, não é apresentado indicadores de avaliação. Os problemas e os exercícios são elaborados por meio de perguntas, não sendo configurados por número de questões ou itens.

\section{CRITÉRIO TECNOLÓGICO-TEÓRICO}

A respeito do bloco tecnológico-teórico de LDMAT, ora é inexistente, ora é definido que: o bit é a menor unidade de medida de informação, a menor unidade de informação com que os computadores trabalham; o bit pode possuir dois valores ( 0 ou 1) e que representam, respectivamente dois estados, não, desligado e sim, ligado; o byte é a combinação de 8 bits ou conjunto de 1 caractere; as unidades de medida de informação, kilobyte, megabyte, gigabyte, entre outras, são enquadradas como múltiplos do byte e que fazem parte do sistema métrico decimal; as velocidades de processamento de dados, megahertz e gigahertz, são definidas por ciclos por segundos. Há, portanto, em LDMAT, superficialidade na abordagem de unidades de medida da informática e de sistema de numeração binário. Em comparação com o bloco tecnológico-teórico de LDCC, neste, há justificativa e explicação de técnicas empregadas por números binários; é mais amplo e aprofundado do que em LDMAT: 
1. Faz-se uma correspondência entre os lados de um cartão (um lado perfurado e o outro não) e os números binários (respectivamente 1e 0), e a partir de sua manipulação (virar cartões - técnica), pode identificar e corrigir erros de códigos por meio de organização de bits em linhas e colunas imaginárias, e acréscimo de bits de paridade para cada linha e coluna, o que "simula" uma técnica de detecção e correção de erros de dados de/por um computador;

2. Um bit é representado na memória principal do computador por um transistor, que pode estar ligado (1) ou desligado (0), ou um capacitor, que pode estar carregado (1) ou descarregado (0);

3. Em relação as transmissões de dados por uma linha telefônica ou enlace de rádio, tons de alta e baixa frequências são utilizados para os uns e zeros (binários);

4. Em discos magnéticos, os bits são representados pela direção de um campo magnético sobre uma superfície revestida, podendo ser norte-sul ou sul-norte, tal que esses artefatos tecnológicos digitais armazenam bits de forma ótica, parte da superfície corresponde a um ou a zero bit a depender se há reflexão de luz (1) ou não (0);

5. Em imagens de computadores, que são formadas por pixels, os binários, 1 ou 0 , representam pontos pretos ou brancos ou grupos de pixels em imagens coloridas;

6. Apresenta técnicas de compressão de dados baseado na manipulação de códigos binários;

7. Baseado em perguntas que tenham como respostas, apenas sim (1) ou não (0), e a partir desta regra, introduz e justifica uma técnica de quantificação de informações.

A partir daquelas sete representações e contextos didáticos ou práticos sociais, cria-se em LDCC a imagem conceitual de números binários - cada zero ou um como um bit. Principalmente, a ordenação de cartões perfurados, ou não, justapostos da direita para a esquerda introduz, didaticamente, o conceito estrutural de sistema de numeração binário.

As técnicas de detecção e correção de erros de dados por computadores são justificadas e explicadas em LDCC por meio de bits de paridade e pelo déci mo dígito utilizado no International Standard Book Number (ISBN);

O LDCC introduz noção de teoria da informação de Shannon ao justificar e explicar a medição de informação contida em mensagens a partir da quantidade de perguntas realizadas para descobri-la (a quantidade de informação contida na mensagem é mensurada pela dificuldade em descobri-la).

\section{CRITÉRIO DE RELAÇÃO COM OUTROS SABERES OU OUTROS BLOCOS DE CONTEÚDO}

LDMAT apresentam elementos históricos, como a história do computador e do crescimento da capacidade de memória de artefatos tecnológicos como fita cassete, CD e aparelhos de MP3; relaciona as unidades de medida de armazenamento e processamento de dados por meio de elementos da 
informática: downloads, MP3, backup, internet, etc; articula o estudo de unidades de medida da informática com a potenciação, as 4 operações fundamentais, medidas de tempo, velocidade e estatística (média e gráfico). Essa relação com potenciação também é mantida em LDCC, este solicitou criar uma técnica que possibilitasse formar qualquer número natural como composição de somas de potências de base 2. Além disso, LDCC trabalha padrões, ordenamentos ou sequências numéricas relacionadas às potências de base 2 (relação com potenciação). Outra relação, a nosso ver, com matemática, é a detecção e correção de erros de dados que podem ser relacionados ao estudo de plano cartesiano. Portanto, os LDMAT mantém relação com elementos da informática ou computação assim como, o LDCC mantém relação com assuntos da matemática.

\section{SÍNTESE}

Tipos de tarefas

\begin{tabular}{cl}
\hline LDMAT & $\begin{array}{l}\text { 12 tipos de tarefas; } \\
\text { Unidades de medida da informática por meio de múltiplos do } \\
\text { byte e sistema métrico decimal. } \\
\\
20 \text { tipos de tarefas; }\end{array}$ \\
LDCC & $\begin{array}{l}\text { Representar, codificar/decodificar, comprimir, detectar/corrigir } \\
\text { erros de dados e medir informação. }\end{array}$ \\
& $\begin{array}{l}\text { Converter um número representado no sistema binário em um } \\
\text { número representado no sistema decimal; }\end{array}$ \\
Elementos & $\begin{array}{l}\text { Representar um número natural como uma soma de potências } \\
\text { de base } 2 ; \\
\text { comuns }\end{array}$ \\
& Determinar a capacidade de memória de um computador.
\end{tabular}

Técnicas

\begin{tabular}{|c|c|}
\hline LDMAT & $\begin{array}{l}3 \text { técnicas referentes às conversões de números representados } \\
\text { entre o sistema binário e o sistema decimal (vice-versa). } \\
1 \text { técnica para converter número representado no sistema binário } \\
\text { em um número representado no sistema decimal; }\end{array}$ \\
\hline LDCC & $\begin{array}{l}2 \text { técnicas para codificar/decodificar imagens; } \\
1 \text { técnica de compressão de textos; } \\
2 \text { técnicas de detecção e correção de erros de dados; } \\
1 \text { técnica de medição de informação. }\end{array}$ \\
\hline & Tecnologias \\
\hline LDMAT & $\begin{array}{l}1 \text { bité a menor unidade de informação e que pode assumir, apenas, } \\
\text { dois valores: } 0 \text { ou 1; } \\
\text { O byte corresponde a } 8 \text { bits; é igual a um caractere; } \\
\text { Definem a base } 10 \text { e a base } 2 \text { numéricas em medidas de informação }\end{array}$ \\
\hline
\end{tabular}


Justificativas e explicações giram em torno de como podemos representar textos e imagens a partir de números binários e de como armazenar dados em memória de computador.

\section{Teorias}

Apenas uma coleção apresenta noções teóricas de grandezas e de

LDMAT medidas.

LDCC Noções teóricas de medida de informação de Claude Shannon.

\section{Critério de Razão de Ser}

As unidades de medida da informática (armazenamento e processamento de dados) são saberes sociais atuais de caráter prático e relacional à outros saberes e áreas de conhecimento; O advento da informática (e de suas unidades de medida) e de sua

LDMAT utilização nos dias atuais; Os computadores operam utilizando o sistema de numeração binário;

A utilização de códigos binários no mundo da Informática.

Os números binários constituem o lócus da Computação; Os computadores atualmente utilizam os números binários para representar informação;

LDCC Os números binários são dados via armazenamento, transmissão, compressão, codificação/decodificação, detecção e correção de erros.

\section{Critério de Relevância}

O estudo de números binários pode auxiliar a compreensão de potenciação;

LDMAT

O armazenamento e processamento de dados de computador são decorridos graças às unidades de medida da informática.

LDCC

Os números bi nários são assuntos fundamentais para a realização de técnicas de medição de informação, de codificação/decodificação, detecção/correção de erros e compressão de dados.

\section{Critério de Identificação}

\begin{tabular}{cl}
\hline LDMAT & $\begin{array}{l}12 \text { tipos de tarefas, } 3 \text { técnicas claras e } 2 \text { implícitas, } 1 \text { tecnologia e } \\
\text { nenhuma teoria (praxeologia local ou pontual). } \\
\text { LDCC }\end{array}$ \\
$\begin{array}{l}\text { 20 tipos de tarefas, } 7 \text { técnicas, } 5 \text { tecnologias e } 1 \text { princípio de teoria } \\
\text { (praxeologia regional) }\end{array}$ \\
Critério Prático-Técnico \\
LDMAT $\begin{array}{l}\text { Não apresenta ilustração ou técnica para a execução das tarefas; } \\
\text { informática; }\end{array}$
\end{tabular}


Todas as tarefas são solicitadas após explicações introdutórias ao capítulo em seções ou tópicos próprios.

As técnicas são apresentadas por etapas (questionamentos);

LDCC As tarefas e subtarefas são atreladas às técnicas; Trabalha técnicas de compressão, codificação/decodificação e correção de erros de dados e medição de informação.

\section{Critério Tecnológico-Teórico}

É inexistente em sua maioria. Apenas uma coleção aborda, de

LDMAT maneira introdutória, noções teóricas de grandeza e de medida; Algumas coleções se restringem a definir o bit e o byte; A veracidade das técnicas não é justificada e nem explicadas.

Todas as técnicas são justificadas embora nem todas sejam explicadas;

As 3 técnicas de compressão de dados ( 2 de imagem e 1 de texto) são justificadas e é imbricada à técnica;

Explica a estrutura numérica do sistema de numeração binário de maneira atrelada a técnica - emprego de cartões perfurados;

IDCC As técnicas de detecção e correção de erros de dados por computadores são justificadas e explicadas por meio de bits de paridade e pelo décimo dígito utilizado no International Standard Book Number (ISBN);

Introduz noção de teoria da informação de Shannon ao justificar e explicar a medição de informação contida em mensagens a partir da quantidade de perguntas realizadas para descobri-la (a quantidade de informação contida na mensagem é mensurada pela dificuldade em descobri-la).

Critério de Relação com outras áreas de saber

Introduz elementos históricos: como a história do computador e do crescimento da capacidade de memória de artefatos tecnológicos como fita cassete, CD e aparelhos de MP3;

Relaciona as unidades de medida de armazenamento e

LDMAT processamento de dados por meio de elementos da informática: downloads, MP3, backup, internet, conexão, etc;

Articula o estudo de unidades de medida da informática com a potenciação, as 4 operações fundamentais, medidas de tempo, velocidade e estatística (média e gráfico).

Identificação de padrões, ordenamentos ou sequências numéricas

LDCC relacionadas às potências de base 2 (Relação com Potenciação); A detecção e correção de erros de dados podem ser relacionados ao estudo de plano cartesiano.

\section{CONCLUSÃO}


Concluímos que as abordagens de números binários em LDCC e em LDMAT são distintas, porém mantêm alguns tipos de tarefas em comum:

1. Converter um número representado no sistema binário em um número representado no sistema decimal;

2. Representar um número natural como uma soma de potências de base 2;

3. Determinar a capacidade de memória de um computador.

Os LDMAT e o LDCC destacam razões comuns para se estudar números binários em livro didático escolar:

1. Os computadores operam utilizando o sistema de numeração binário;

2. A utilização, atual, de códigos binários no mundo da Informática;

3. $O$ advento da informática (unidades de medida) e de sua utilização nos dias atuais;

A abordagem de números binários em LDCC é mais ampla que em LDMAT porque:

1. Os números binários são trabalhados como suporte organizacional para representar, comprimir, armazenar e detectar dados, assim como corrigir seus erros. Além disso, mantém como proposta didática trabal har atividades voltadas para a introdução de elementos conceituais de ciência da computação, como algoritmo, grafos, etc;

2. É configurada como uma praxeologia regional, enquanto que em LDMAT é configurada como praxeologias: pontual ou local;

A abordagem de números binários em LDCC é mais aprofundada que em LDMAT porque:

1. Existe a explicação estrutural, funcional do sistema de numeração binário por explicar, por exemplo, que um zero à direita de um algarismo binário, significa que o número, formado por estes algarismos, é dobrado, justificando desta forma o porquê de ser definido na base 2 , procedimento semelhante a organização estrutural do sistema de numeração decimal que no caso de acréscimo de um zero à direita, equivale a multiplicação do número por 10 , justificando desta forma o porquê de ser definido na base 10.

A abordagem de números binários em LDCC é, portanto, intracomputação (embora seja, em partes, contextualizado por conceitos da matemática), enquanto que a abordagem de sistema de numeração binário em LDMAT é, em sua maioria, proposta de maneira isolada, mantendo-se como um tema de curiosidade no Manual do Professor ao invés de um tema para a "formação matemática". Apenas uma coleção trabal ha o sistema de numeração em primeiro plano, trabalha duas técnicas de conversões de números representados entre os sistemas decimal e binário (vice-versa). No entanto, mantém um único tipo de tarefa: converter um número representado no sistema binário em um número representado no sistema decimal.

As tarefas referentes aos números binários em LDCC são atreladas à exercitação de técnicas e algumas delas são justificadas e fundamentadas teoricamente, como o caso da fundamentação teórica de medidas de informação 
pela teoria da informação e comunicação de Shannon. A maioria das tarefas é direcionada à execução de determinada etapa da técnica. Já em LDMAT, as tarefas são solicitadas após uma breve introdução do assunto.

Em relação às propostas didáticas de unidades de medidas da informática (unidades de medida de armazenamento e processamento de dados) em LDMAT e em LDCC também são distintas:

1. Todos os LDMAT trabalham os múltiplos de byte (quilobyte, megabyte, gigabyte, terabyte) e são definidas no si stema decimal. Já o LDCC, apenas, trabalha o bit e o byte, e são definidos no sistema numérico binário; não trabalha a velocidade de processamento de dados;

2. Quase todos os LDMAT mantêm uma proposta de trabalhar o tema como um conteúdo auxiliar, de suporte ou de contexto ao tema de estudo: potenciação. Já em LDCC, o bit e o byte constituem pré-requisitos para a compreensão de medições de informação, de codificação/decodificação de dados, de compressão de dados e de deteç̧ão e correção de erros de dados (temas e assuntos da própria computação);

3. São abordados em LDMAT, de maneira contextual, por elementos e dispositivos da Informática (internet, tablets, pen drives, máquinas fotográficas digitais, computadores, entre outros) e por abordagem superficial a respeito da história do computador e de outros dispositivos tecnológicos. Em relação à abordagem em LDCC, o estudo do tema é básico para a aprendizagem de medida de informação. 


\title{
Binary numbers in mathematics and computation textbooks: a comparation
}

\begin{abstract}
It is Based on the identification of approach of binary numbers in computation and mathematics textbooks at Elementary School, its objective is to compare this approach based on the praxeology and its criteria of evaluation (elements of the Anthropological Theory of the Didactic). At first, the comparation is made through praxeological elements: types of task, technique, technology and theory. Then, the comparation is made through praxeological evaluation criteria: criterion of reason of being, criterion of relevance, criterion of identification, practical-technical criterion, technological-theoretical criterion and criterion of relation with other knowledge. It realizes that the approach of binary numbers in computation textbook is broader and deeper than in all mathematics textbooks in Elementary School evaluated by PNLD 2014.
\end{abstract}

KEYWORDS: Binary numbers. Mathematics textbook. Computation textbook. 


\section{REFERÊNCIAS}

ANDRINE, Á.; VASCONCELLOS, M. J. C. Praticando matemática. 3. ed. São Paulo: Editora do Brasil, v. 6, 2012.

BELL, TIM. Computer Science Unplugged: ensinando computação sem o uso do computador. Tradução de Luciano Porto Barreto. [S.I.]: csunplugged.org, 2011. $198 \mathrm{p.}$

BOSCH, M.; GASCÓN, J. 25 años de Transposición Didáctica. In: L. RUIZ-HIGUERAS, A. E. F. J. G. (. ). Sociedad, Escuela y Matemáticas. Aportaciones de la Teoría Antropológica de lo Didáctico. In L. Ruiz-Higueras, A. Estepa, F. J. García: [s.n.], 2007. p. 385-406.

CHEVALLARD, Y. Analyse des pratiques enseignates et didactique des mathematiques: lápproche antropologique, 1998. Disponivel em: <http://yves.chevallard.free.fr>. Acesso em: 11 abril 2014.

DANTE, L. R. Projeto Teláris: Matemática. 1. ed. São Paulo: Ática, v. 6, 2012.

GLASER, A. History of Binary and other nondecimal numeration. [S.I.]:Tomash Publishers, 1981.

MENDES, H. D. L. Os números binários nas instituições transpositivas: o caso das diretrizes curriculares. XVIII EBRAPEM, Recife, 2014.

MENDES, H. D. L. Análise praxeológica de livro didático de matemática referente ao estudo de números binários. REVEMAT, Florianópolis, 10, n. 1, dezembro 2015. 199-219.

MENDES, H. D. L. Análise praxeológica de livro didático de computação referente ao estudo de números binários. Revista Brasileira de Ensino de Ciências e Tecnologia, 9, n. 3, 2016a. 311-333.

MENDES, H. D. L. Transposição didática: o caso das unidades de memória da informática. REVEMAT, 11, n. 2, 2016b. 156-172.

MENDES, H. D. L. Os números binários: do saber escolar ao saber científico. Jornal Internacional de Estudos em Educação Matemática, prelo 2017a. 
MENDES, H. D. L. Análise praxeológica de livros didáticos de matemática: o caso dos números binários. Educação Matemática Pesquisa, prelo 2017b.

SILVA, V. V. D. Números: construções e propriedades. Goiânia: UFG, 2003.

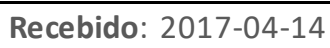
computação: uma comparação. Revista Brasileira de Ensino de Ciência e Tecnologia, v. 11, n. 1, 2018. Disponível em:

$<$ https://periodicos.utfpr.edu.br/rbect/article/view/5801>. Acesso em: xxx. Correspondência: Herman do Lago Mendes - herman2000@zipmail.com.br Direito autoral: Este artigo está licenciado sob os termos da Licença Creative Commons-Atribuição 4.0 Internacional.

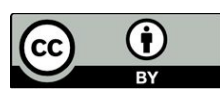

